\title{
ON THE NUMERICAL CLASSIFICATION OF THE SINGULARITIES OF ROBOT MANIPULATORS
}

\author{
Oriol Bohigas $^{1}$, Dimiter Zlatanov ${ }^{2}$, Montserrat Manubens ${ }^{1}$, and Lluís Ros ${ }^{1}$ \\ ${ }^{1}$ Institut de Robòtica i Informàtica Industrial (CSIC-UPC) \\ 08028 Barcelona, Catalonia \\ \{obohigas,mmanuben,Ilros\}@iri.upc.edu \\ ${ }^{2}$ PMAR Laboratory (DIMEC) \\ Università di Genova \\ 16145 Genoa, Italy \\ zlatanov@dimec.unige.it
}

\begin{abstract}
This paper is concerned with the task to obtain a complete description of the singularity set of any given non-redundant manipulator, including the identification and the precise computation of each constituent singularity class. Configurations belonging to the same class are equivalent in terms of the various types of kinematic and static degeneracy that characterize mechanism singularity. The proposed approach is an extension of recent work on computing singularities using a numerical method based on linear relaxations. Classification is sought by means of a hierarchy of singularity tests, each formulated as a system of quadratic or linear equations, which yields sets of classes to which an identified singularity cannot belong. A planar manipulator exemplifies the process of classification, and illustrates how, while most singularities get completely classified, for some lower-dimensional subsets one can only identify a restricted list of possible singularity classes.
\end{abstract}

\section{INTRODUCTION}

The study of robot singularities is a central topic in Robot Kinematics because the properties of the manipulator change dramatically, and often different kinds of problems may appear [1]. Even though the literature on this topic is copious, an approach to obtain a complete description of the singularity set of any non-redundant manipulator has not yet been given. Such an approach should include the identification and precise computation of each constituent singularity class. Most works, including previous methods for computing singularities, focus on particular classes of singularities, and on specific robot architectures [2-14].
In [15], a general singularity classification was attempted, based on an input-output velocity equation. This overlooks cases where the motion of the mechanism cannot be described with the input and output speeds only. In [16,17] more general methods from differential geometry were applied, and three singularity types were proposed. In $[18,19]$, a general manipulator model, in terms of differentiable mappings between manifolds, allowed a rigorous mathematical definition of kinematic singularity. Three basic kinds of singularities were identifiedconfiguration-space, input, and output singularities-but the approach also allowed for a finer classification using six types, each of which corresponds to a different kinematic phenomenon that may occur in the singularity. It was also shown that a singularity always belongs to several of these types simultaneously, but that only twenty-one type combinations, or classes, can exist. Thus, a manipulator can have at most twenty-one singularity classes each containing configurations equivalent in terms of the occurring types of kinematic and static degeneracy. Various conditions for the presence of singularities of all types have been presented $[19,20]$. Recent work uses them to obtain an exhaustive computation of the whole singularity set, and each of the six singularity types, by means of a numerical method based on linear relaxations [21]. However, an efficient method for the complete classification of the singularity set of a given mechanism, i.e. the identification of the nonintersecting singularity classes, is still to be achieved.

The present work aims at completing the task of exhaustive classification of the singularity set of an arbitrary non-redundant manipulator by extending the work in [21]. The paper shows that, for a singular configuration, a sequence of tests allows determining groups of classes to which the configuration cannot belong. 
This fact allows defining a process that is able to precisely classify most singularities, and only in rare cases this test is subject to sufficient numerical precision.

The rest of the paper is organized as follows. Section "Preliminaries" provides some necessary background, including two systems of equations that allow identifying the whole singularity set as well as the description of the various singularity types and classes. Section "Isolation of the Singularity Set" describes a numerical method that is able to solve the previous systems, thus isolating the complete singularity set of the given manipulator. Section "Classification of the Singularity Set" provides an approach to classify the obtained singularity set by applying the same method on additional systems of equations. The performance of the approach is illustrated in Section "Illustrative Example".

\section{PRELIMINARIES}

Every manipulator configuration can be described by a vector $\boldsymbol{q}$ of scalar generalized-coordinate variables. In the case of manipulators containing closed-loops, or when a joint does not admit a global parametrization, not all values of $\boldsymbol{q}$ correspond to feasible configurations, and the configuration space is given by the solution set of a system of non-linear equations

$$
\boldsymbol{\Phi}(\boldsymbol{q})=\mathbf{0},
$$

which expresses the assembly constraints imposed by the joints [22]. In addition, the possible motion rates of the manipulator, i.e. its feasible instantaneous motions, are characterized by a system of linear equations

$$
\boldsymbol{L} \cdot \boldsymbol{m}=\mathbf{0}
$$

called the velocity equation in [19]. The matrix $\boldsymbol{L}$ depends on the configuration $\boldsymbol{q}$. The velocity vector $\boldsymbol{m}=\left[\boldsymbol{\Omega}^{o \top}, \boldsymbol{\Omega}^{a \top}, \boldsymbol{\Omega}^{p \top}\right]^{\top}$ has as components $\boldsymbol{\Omega}^{o}$, a vector describing the rate of change of the output motion (for example, the velocity of a point or the angular velocity, or the twist, of an end-effector body), as well as $\boldsymbol{\Omega}^{a}$ and $\boldsymbol{\Omega}^{p}$, describing the rate of change of input and passive motion, respectively (typically, $\boldsymbol{\Omega}^{a}$ and $\boldsymbol{\Omega}^{p}$ are the actuated and unactuated joint speeds, respectively). Such a system of equations can be obtained for any manipulator, for instance using the twist loop equations, and therefore can be used for the practical identification of singularities.

In this paper we assume that, for every configuration, the dimension of the output and input velocity vectors are equal to the (global) mobility, $n$, of the kinematic chain (the mobility is equal to the dimension of the configuration space, i.e., to the maximum dimension of its tangent space, wherever such space exists [23]). For later use, assume that $\boldsymbol{L}$ has $N$ rows, and thus it is a $N \times(N+n)$ matrix, and let $\boldsymbol{L}_{I}, \boldsymbol{L}_{O}$ and $\boldsymbol{L}_{P}$ be the submatrices of $\boldsymbol{L}$ obtained by removing the columns corresponding to the input, output, and both the input and output, respectively.

\section{Identification of the Singularity Set}

The instantaneous kinematic analysis of a manipulator addresses two main problems:

The forward instantaneous kinematics problem (FIKP): find $\boldsymbol{m}$ given the input $\boldsymbol{\Omega}^{a}$, and

The inverse instantaneous kinematics problem (IIKP): find $\boldsymbol{m}$ given the output $\boldsymbol{\Omega}^{o}$.

Note that in each case it is required to find all instantaneous parameters of the manipulator, not just the output or input speeds, respectively. Following [19], a configuration is said to be nonsingular when both the FIKP and the IIKP have unique solutions for any input or output. This leads to the identification of three basic types of singularities, namely, input singularities and output singularities, where the FIKP and the IIKP are undetermined, respectively, and configuration-space singularities, where both the FIKP and IIKP become undetermined for any definition of input or output from the given velocity variables.

As proven in $[19,20]$, the singularities of a manipulator can be characterized by those configurations where either the matrix $\boldsymbol{L}_{I}$ or the matrix $\boldsymbol{L}_{O}$ is rank deficient. Note that if a matrix is rank deficient, its kernel has to be non-null and, in particular, it must include a vector of unit norm. Thus, all singularities can be determined by solving the following two systems of equations:

$$
\left.\left.\begin{array}{c}
\boldsymbol{\Phi}(\boldsymbol{q})=\mathbf{0} \\
\boldsymbol{L}_{I}^{\top} \boldsymbol{\xi}=\mathbf{0} \\
\|\boldsymbol{\xi}\|^{2}=1
\end{array}\right\}, \begin{array}{c}
\boldsymbol{\Phi}(\boldsymbol{q})=\mathbf{0} \\
\boldsymbol{L}_{O}^{\top} \boldsymbol{\xi}=\mathbf{0} \\
\|\boldsymbol{\xi}\|^{2}=1
\end{array}\right\}
$$

The first equation of each system constrains $\boldsymbol{q}$ to be a feasible configuration of the mechanism. The second and third equations imply the existence of a non-zero vector in the kernel of the corresponding matrix. Note that $\|\boldsymbol{\xi}\|$ can be any consistent norm; for instance, we may use $\|\boldsymbol{\xi}\|^{2}=\boldsymbol{\xi}^{\top} \boldsymbol{D} \boldsymbol{\xi}$ with $\boldsymbol{D}$ a diagonal matrix whose entries have the proper physical units. There is no need for the norm to be invariant with respect to change of frame or units, because the condition $\|\boldsymbol{\xi}\|^{2}=1$ only serves to guarantee that $\boldsymbol{\xi}$ is not $\mathbf{0}$.

The solution of the system on the left in Eq. (2) corresponds to all configurations where the FIKP is undeterminate, including all input singularities, while the solution of the system on the right includes all output singularities, where the IIKP is undeterminate. Configuration-space singularities will satisfy both systems in (2), as well as any analogous system obtained by deleting $n$ columns in $\boldsymbol{L}$. The whole singularity set can be computed as the union of the sets obtained as solutions of each system. 


\section{Types and Classes of Singularities}

The singularity set can be seen to contain six distinct lowlevel singularity types, depending on the cause of the degeneracy of the FIKP or IIKP. These are redundant input (RI), redundant output (RO), impossible input (II), impossible output (IO), increased instantaneous mobility (IIM) and redundant passive motion (RPM) [19].

Each of the six singularity-type definitions describes an important change in the kinematic properties of the manipulator that occurs in a singular configuration of that type. When the mechanism is in a singularity of type RO or IO (RI or II), the output (input) is undeterminate or restricted. In an IIM-type configuration the instantaneous motion of the manipulator is undeterminate, no matter which $n$ parameters are being controlled. In an RPM-type singularity, the passive motion of the mechanism is undeterminate, which may create problems such as interference with other links and obstacles.

Moreover, as proved in [19], any singular configuration belongs to several of the six singularity types, but only twenty-one different combinations of singularity types, called singularity classes, are possible. These correspond to the cells marked with "Y" in Table 1. Configurations belonging to the same class are equivalent in terms of the various types of kinematic and static degeneracy that characterize mechanism singularity. It is, therefore, desirable to identify each constituent singularity class in order to obtain a complete description of the singularity set. To see how such identification can be performed, we next recall a method for computing the whole singularity set [21], and then show how such method can be applied to classify the points of the set into the various possible singularity classes.

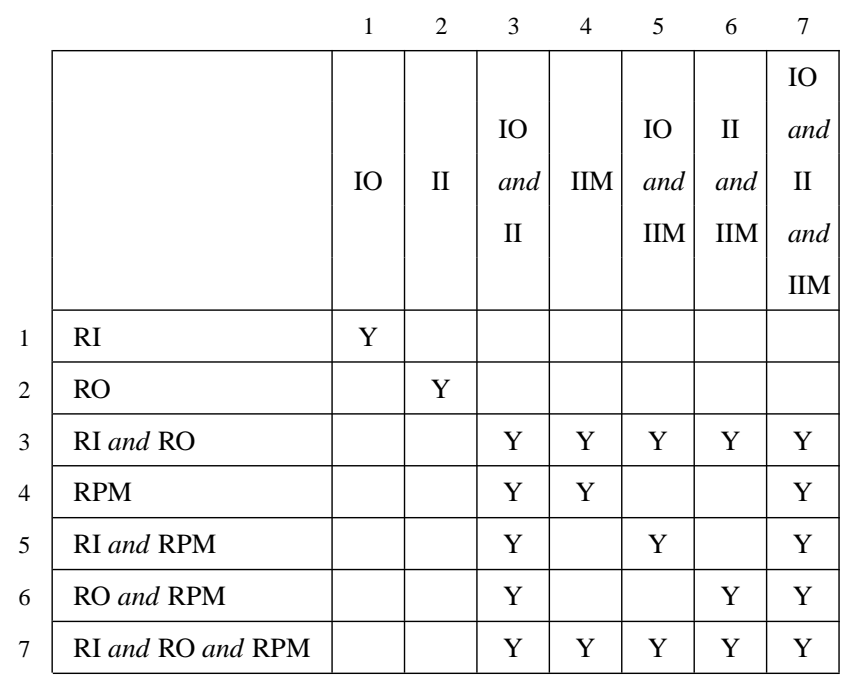

TABLE 1. The twenty-one singularity classes.

\section{ISOLATION OF THE SINGULARITY SET}

The method, which is based on an earlier approach for the position analysis of multi-loop linkages [24], consists in first formulating the systems of equations in (2) in an appropriate way, and then using a numerical technique that exploits the particular structure of these systems to isolate the singularity set at the desired resolution.

\section{Equation formulation}

For manipulators with non-helical pairs, and departing from the generalised coordinates $\boldsymbol{q}$ proposed in [24], it is possible to formulate both systems in (2) so that they adopt the form of a polynomial system of quadratic equations, i.e. equations where only monomials of the form $a, a^{2}$, or $a b$ intervene, where $a$ and $b$ refer to any two of the variables. All variables in such systems will only take values within limited intervals, because interval bounds for all of the $\boldsymbol{q}$ variables can be readily obtained [24], and equation $\|\boldsymbol{\xi}\|^{2}=1$ limits the components of $\boldsymbol{\xi}$ to the $[-1,1]$ range. This allows the use of a particularly simple technique to compute the solution of the systems in (2) numerically. For ease of explanation, we will write any one of these systems as

$$
\boldsymbol{F}(\boldsymbol{y})=\mathbf{0}
$$

hereafter, where $\boldsymbol{y}$ is the vector of variables involved in the system, and $\boldsymbol{F}(\boldsymbol{y})$ is a quadratic vector-valued function.

\section{Numerical solution}

In order to solve (3), we start defining the changes of variables $p_{i}=y_{i}^{2}$ and $b_{k}=y_{i} y_{j}$ for each quadratic and bilinear monomial in (3), transforming the system into the expanded form

$$
\left.\begin{array}{l}
\boldsymbol{\Lambda}(\boldsymbol{x})=\mathbf{0} \\
\boldsymbol{\Gamma}(\boldsymbol{x})=\mathbf{0}
\end{array}\right\}
$$

where $\boldsymbol{\Lambda}(\boldsymbol{x})=\mathbf{0}$ is a collection of linear equations in $\boldsymbol{x}$ and $\boldsymbol{\Gamma}(\boldsymbol{x})=\mathbf{0}$ is a collection of quadratic equations, each of which adopts one of the two forms $x_{k}=x_{i}^{2}$ or $x_{k}=x_{i} x_{j}$. The vector of variables $\boldsymbol{x}$ contains the previous $\boldsymbol{y}$ variables and the newly defined ones $p_{i}$ and $b_{k}$. Note that all variables in $\boldsymbol{x}$ are bounded within limited intervals, because the $\boldsymbol{y}$ variables are. Thus, from the Cartesian product of such intervals, one can define a box $\mathscr{B}$ that initially bounds all solutions of the considered system.

We can now solve a system of the form of (4) by recursively applying two operations on the initial box $\mathscr{B}$, called box shrinking and box splitting. Using box shrinking, portions of $\mathscr{B}$ containing no solution are eliminated by narrowing some of its defining intervals. This process is repeated until either the box is reduced to an empty set, in which case it contains no solution, 

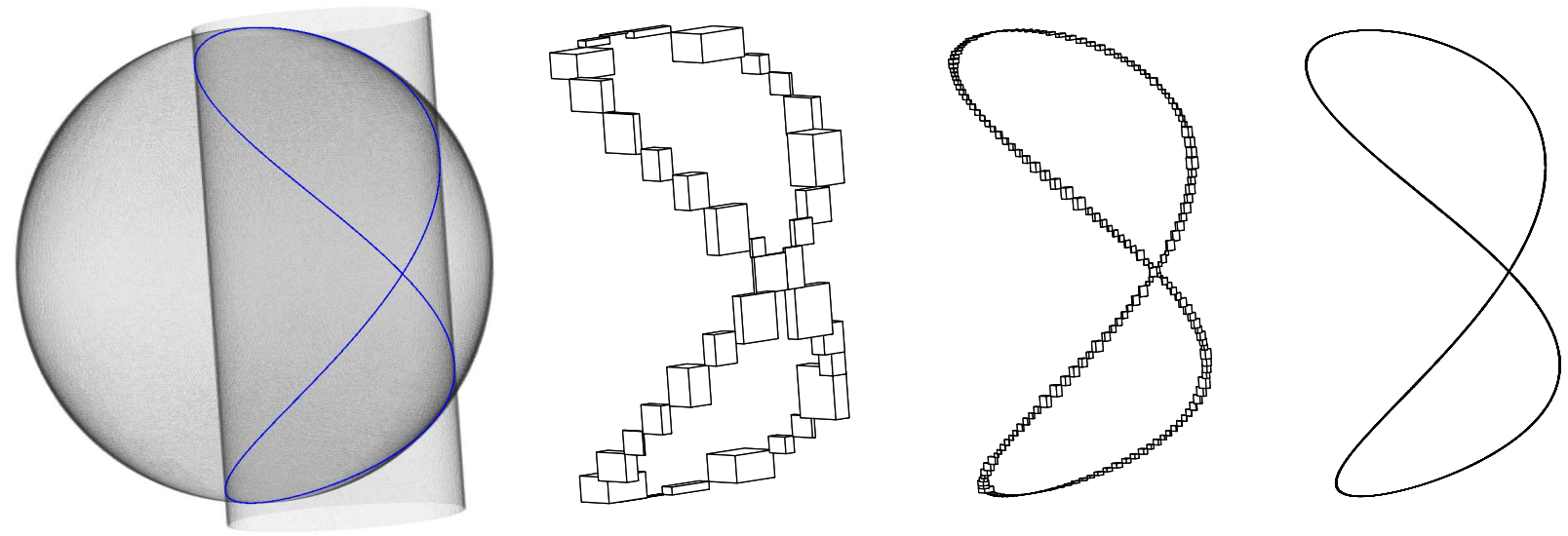

FIGURE 1. From left to right: Viviani's curve, solution of the system formed by equations $x^{2}+y^{2}+z^{2}=4 a^{2}$ and $(x-a)^{2}+y^{2}=a^{2}$, and three steps of the progression of the numerical method when computing such curve.

or the box is "sufficiently" small, in which case it is considered a solution box, or the box cannot be "significantly" reduced, in which case it is bisected into two sub-boxes via box splitting (which simply bisects its largest interval). To converge to all solutions, the whole process is recursively applied to the new subboxes, until one obtains a collection $B$ of solution boxes whose side lengths are below a given threshold $\sigma$. As an illustrative example, the progression of the method is illustrated in Fig. 1.

The crucial operation in this scheme is box shrinking, which is implemented as follows. Note first that the solutions falling in some sub-box $\mathscr{B}_{c} \subseteq \mathscr{B}$ must lie in the linear variety defined by $\boldsymbol{\Lambda}(\boldsymbol{x})=0$. Thus, we may shrink $\mathscr{B}_{c}$ to the smallest possible box bounding this variety inside $\mathscr{B}_{c}$. The limits of the shrunk box along, say, dimension $x_{i}$ can be found by solving the two linear programs

LP1: Minimize $x_{i}$, subject to: $\boldsymbol{\Lambda}(\boldsymbol{x})=\mathbf{0}, \boldsymbol{x} \in \mathscr{B}_{c}$

$$
\text { LP2: Maximize } x_{i} \text {, subject to: } \mathbf{\Lambda}(\boldsymbol{x})=\mathbf{0}, \boldsymbol{x} \in \mathscr{B}_{c} \text {. }
$$

However, observe that $\mathscr{B}_{c}$ can be further reduced, because the solutions must also satisfy all equations $x_{k}=x_{i}^{2}$ and $x_{k}=x_{i} x_{j}$ in $\boldsymbol{\Gamma}(\boldsymbol{x})=\mathbf{0}$. These equations can be taken into account by noting that, if $\left[v_{i}, u_{i}\right]$ denotes the interval of $\mathscr{B}_{c}$ along dimension $x_{i}$, then:

1. The portion of the parabola $x_{k}=x_{i}^{2}$ lying inside $\mathscr{B}_{c}$ is bound by the triangle $A_{1} A_{2} A_{3}$, where $A_{1}$ and $A_{2}$ are the points where the parabola intercepts the lines $x_{i}=v_{i}$ and $x_{i}=u_{i}$, and $A_{3}$ is the point where the tangent lines at $A_{1}$ and $A_{2}$ meet (Fig. 2a).

2. The portion of the hyperbolic paraboloid $x_{k}=x_{i} x_{j}$ lying inside $\mathscr{B}_{c}$ is bound by the tetrahedron $B_{1} B_{2} B_{3} B_{4}$, where the points $B_{1}, \ldots, B_{4}$ are obtained by lifting the corners of the rectangle $\left[v_{i}, u_{i}\right] \times\left[v_{j}, u_{j}\right]$ vertically to the paraboloid (Fig. 2b).
Thus, linear inequalities corresponding to these bounds can be added to LP1 and LP2, which usually produces a much larger reduction of $\mathscr{B}_{c}$ or, if one of the linear programs is found unfeasible, its complete elimination.

The collection $B$ of all solution boxes, which is returned as output upon termination, is said to form a box approximation of the solution set of Eq. (4), because the boxes in $B$ form a discrete envelop of such set, whose accuracy can be adjusted through the $\sigma$ parameter. Notice that the algorithm is complete, in the sense that it will succeed in isolating all solution points of Eq. (4) accurately, provided that a small-enough value for $\sigma$ is used. Detailed properties of the algorithm, including an analysis of its completeness, correctness, and convergence order, are given in [24].

(a)

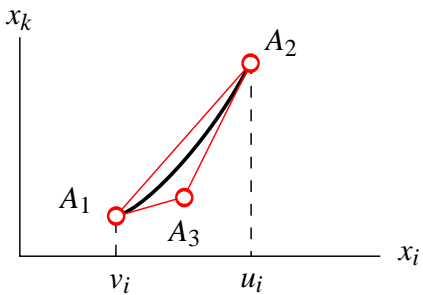

(b)

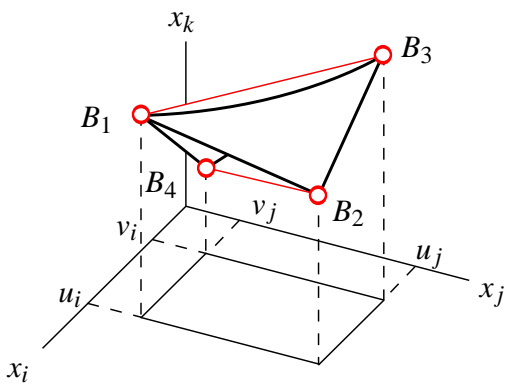

FIGURE 2. Polytope bounds within box $\mathscr{B}_{c}$. 


\section{CLASSIFICATION OF THE SINGULARITY SET}

The union of the collection of boxes obtained for each system in (2) gives a complete representation of the whole singularity set of the manipulator. Its classification among singularity classes can then be sought by applying a hierarchy of singularity tests on each box which yields sets of classes that cannot be present inside such box.

As it will be seen, the process is able to precisely classify most of the singularities, and only exceptionally this is subject to sufficient numerical precision. In the latter cases, if box splitting does not have the necessary resolution, for some boxes there will remain a few singularity classes, for which the procedure can neither confirm nor deny the presence of some of their configurations in the box.

\section{Singularity Tests}

The following propositions, proved in [19], give the conditions for the occurrence of the different singularity types in function of the rank of matrix $\boldsymbol{L}$ and its submatrices $\boldsymbol{L}_{I}, \boldsymbol{L}_{O}$ and $\boldsymbol{L}_{P}$ :

(i) $\boldsymbol{q} \in\{R I\} \Longleftrightarrow \operatorname{rank} \boldsymbol{L}_{O}<\operatorname{rank} \boldsymbol{L}_{p}+n$,

(ii) $\boldsymbol{q} \in\{R O\} \Longleftrightarrow \operatorname{rank} \boldsymbol{L}_{I}<\operatorname{rank} \boldsymbol{L}_{p}+n$,

(iii) $\boldsymbol{q} \in\{R P M\} \Longleftrightarrow \operatorname{rank} \boldsymbol{L}_{p}<N-n$,

(iv) $\boldsymbol{q} \in\{I I\} \Longleftrightarrow \operatorname{rank} \boldsymbol{L}_{I}<\operatorname{rank} \boldsymbol{L}$,

(v) $\boldsymbol{q} \in\{I O\} \Longleftrightarrow \operatorname{rank} \boldsymbol{L}_{O}<\operatorname{rank} \boldsymbol{L}$

(vi) $\boldsymbol{q} \in\{I I M\} \Longleftrightarrow \operatorname{rank} \boldsymbol{L}<N$,

(vii) $\boldsymbol{q} \in\{R I\}$ or $\boldsymbol{q} \in\{R P M\} \Longleftrightarrow \boldsymbol{q} \in\{I O\}$ or $\boldsymbol{q} \in\{I I M\} \Longleftrightarrow \boldsymbol{L}_{O}$ is singular,

(viii) $\boldsymbol{q} \in\{R O\}$ or $\boldsymbol{q} \in\{R P M\} \Longleftrightarrow \boldsymbol{q} \in\{I I\}$ or $\boldsymbol{q} \in\{I I M\} \Longleftrightarrow \boldsymbol{L}_{I}$ is singular

These propositions allow to systematically define systems of equations that can be used as tests to discard the occurrence of a class or particular singularity types inside a given box $\mathscr{B}_{i}$ by imposing the appropiate rank deficiency of the corresponding matrices. The basic tests are given in Table 2, and more complex ones can be derived by joining the equations of two or more tests of the table.

The simplest tests, labeled T1 and T2, follow from propositions (iii) and (vi). Indeed, as $\boldsymbol{L}_{p}$ is a $N \times(N-n)$ matrix, if T1 contains no solutions for a given box $\mathscr{B}_{i}$, one can be sure that there are no RPM-type singular configurations in such box because the kernel of $\boldsymbol{L}_{p}$ does not contain any non-zero vector for any configuration inside $\mathscr{B}_{i}$, i.e. it is full rank everywhere. Thus, proposition (iii) is not satisfied. Equivalently, T2 allows to discard the occurrence of IIM-type singularities inside $\mathscr{B}_{i}$ if it has no solutions. Additionally, it is possible to discard any singularity corresponding to the (RPM, IIM) class (row 4, column 4 of Table 1) if the system resulting of combining the equations of T1 and $\mathrm{T} 2$ has no solution.

Tests T3 to T6 have a similar structure; we impose the rank deficiency of two matrices and that the vectors in the kernels are different by making them orthogonal to each other. To under-

\begin{tabular}{|c|c|c|c|}
\hline Test name & Propositions & Test equations & $\begin{array}{c}\text { Singularity types } \\
\text { involved }\end{array}$ \\
\hline $\mathrm{T} 1$ & (iii) & $\left.\begin{array}{r}\boldsymbol{\Phi}(\boldsymbol{q})=\mathbf{0} \\
\boldsymbol{L}_{P} \boldsymbol{\xi}_{P}=\mathbf{0} \\
\left\|\boldsymbol{\xi}_{P}\right\|^{2}=1\end{array}\right\}$ & RPM \\
\hline $\mathrm{T} 2$ & (vi) & $\begin{array}{r}\boldsymbol{\Phi}(\boldsymbol{q})=\mathbf{0} \\
\boldsymbol{L}^{\top} \boldsymbol{\xi}_{L}=\mathbf{0} \\
\left\|\boldsymbol{\xi}_{L}\right\|^{2}=1\end{array}$ & IIM \\
\hline $\mathrm{T} 3$ & (i) and (iii) & $\begin{array}{c}\boldsymbol{\Phi}(\boldsymbol{q})=\mathbf{0} \\
\boldsymbol{L}_{O} \boldsymbol{\xi}_{O}=\mathbf{0} \\
\boldsymbol{L}_{P} \boldsymbol{\xi}_{P}=\mathbf{0} \\
\left\|\boldsymbol{\xi}_{O}\right\|^{2}=\left\|\boldsymbol{\xi}_{P}\right\|^{2}=1 \\
{\left[\boldsymbol{\xi}_{P} \mathbf{0}\right]^{\top} \boldsymbol{\xi}_{O}=0}\end{array}$ & RI and RPM \\
\hline $\mathrm{T} 4$ & (ii) and (iii) & $\begin{array}{c}\boldsymbol{\Phi}(\boldsymbol{q})=\mathbf{0} \\
\boldsymbol{L}_{I} \boldsymbol{\xi}_{I}=\mathbf{0} \\
\boldsymbol{L}_{P} \boldsymbol{\xi}_{P}=\mathbf{0} \\
\left\|\boldsymbol{\xi}_{I}\right\|^{2}=\left\|\boldsymbol{\xi}_{P}\right\|^{2}=1 \\
{\left[\boldsymbol{\xi}_{P} \mathbf{0}\right]^{\top} \boldsymbol{\xi}_{I}=0}\end{array}$ & RO and RPM \\
\hline T5 & (iv) and (vi) & $\begin{array}{c}\boldsymbol{\Phi}(\boldsymbol{q})=\mathbf{0} \\
\boldsymbol{L}_{I}^{\top} \boldsymbol{\xi}_{I}=\mathbf{0} \\
\boldsymbol{L}^{\top} \boldsymbol{\xi}_{L}=\mathbf{0} \\
\left\|\boldsymbol{\xi}_{I}\right\|^{2}=\left\|\boldsymbol{\xi}_{L}\right\|^{2}=1 \\
\boldsymbol{\xi}_{I}^{\top} \boldsymbol{\xi}_{L}=0\end{array}$ & II and IIM \\
\hline T6 & (v) and (vi) & $\begin{array}{c}\boldsymbol{\Phi}(\boldsymbol{q})=\mathbf{0} \\
\boldsymbol{L}_{O}^{\top} \boldsymbol{\xi}_{O}=\mathbf{0} \\
\boldsymbol{L}^{\top} \boldsymbol{\xi}_{L}=\mathbf{0} \\
\left\|\boldsymbol{\xi}_{O}\right\|^{2}=\left\|\boldsymbol{\xi}_{L}\right\|^{2}=1 \\
\boldsymbol{\xi}_{O}^{\top} \boldsymbol{\xi}_{L}=0\end{array}$ & IO and IIM \\
\hline $\mathrm{T} 7$ & (vii) & $\left.\begin{array}{r}\boldsymbol{\Phi}(\boldsymbol{q})=\mathbf{0} \\
\boldsymbol{L}_{O} \boldsymbol{\xi}_{O}=\mathbf{0} \\
\left\|\boldsymbol{\xi}_{O}\right\|^{2}=1\end{array}\right\}$ & II, IO, RI, RO \\
\hline $\mathrm{T} 8$ & (viii) & $\left.\begin{array}{c}\boldsymbol{\Phi}(\boldsymbol{q})=\mathbf{0} \\
\boldsymbol{L}_{I} \boldsymbol{\xi}_{I}=\mathbf{0} \\
\left\|\boldsymbol{\xi}_{I}\right\|^{2}=1\end{array}\right\}$ & II, IO, RI, RO \\
\hline
\end{tabular}

TABLE 2. Dimension of vectors $\boldsymbol{\xi}_{O}, \boldsymbol{\xi}_{I}$ and $\boldsymbol{\xi}_{L}$ is $N ; \boldsymbol{\xi}_{P}$ is $N-n$.

stand these tests take propositions (iv) and (vi) involved in T5, for instance. It can be seen that for a configuration to be of type II and IIM, both $\boldsymbol{L}_{I}$ and $\boldsymbol{L}$ have to be rank defficient with $\operatorname{rank} \boldsymbol{L}_{I}<\operatorname{rank} \boldsymbol{L}$. Note also that any vector in the kernel of $\boldsymbol{L}^{\top}$ is also in the kernel of $\boldsymbol{L}_{I}^{\top}$. If T5 had solutions inside a box $\mathscr{B}_{i}$, 
there would be a configuration where $\boldsymbol{L}$ loses rank by 1 at least and $\boldsymbol{L}_{I}$ by 2 at least. Thus, if T5 has no solutions we can be sure that there are no singular configurations of type II and IIM in $\mathscr{B}_{i}$. Similar reasoning applies for tests T3, T4 and T6.

The last two systems of equations of Table 2, T7 and T8, can be used to discard classes of singularities only if previous types have been already discarded. Note that this systems are the same as the ones in Eq. (2), that encode the forward and inverse singularities. In fact, if there are no RPM- or IIM-type singularities in a box, these test serve to discard types or classes involving RI or RO or involving IO or II, respectively. This results from propositions (vii) and (viii).

Following the same spirit, one can define tests for most of the possible singularity classes in Table 1 , by properly combining the equations of the tests given in Table 2, in order to discard the maximum possible number of singularity classes for a box $\mathscr{B}_{i}$, as it is explained next.

\section{The Process of Classification}

The process of classification of the singularity locus consists in applying a sequence of singularity tests on each of the boxes in the collection $B$ returned as solution of Eq. (2) by the numerical method. In principle, using sufficient numerical precision, each box contains at least one singular configuration that could belong to any of the 21 possible singularity classes of Table 1 . For each box, the idea of the process is to discard as many classes as possible, until one ends up with only one class being possible or a restricted set of classes that are or may be present inside the box.

Notice that all tests have been defined as systems of linear or quadratic equations, and are thus solvable using the same numerical method described, taking as initial bounding box the exact ranges given by the box $\mathscr{B}_{i}$ being examined. All tests have been defined in a way that, if there is no box as solution of the system, the corresponding singularity types or classes can be discarded.

The hierarchy of tests has three levels, going from the simplest singularities to more complex ones (several types involved):

1. Test RPM- and IIM- type singularities.

2. Test combinations corresponding to rows or columns of Table 1.

3. Test particular singularity classes.

Given the possible singularity classes and provided that IIMand RPM-type singularities are usually lower dimensional sets with respect to the whole singularity set, it is reasonable to first test the occurrence of these singularity types using systems $\mathrm{T} 1$ and $\mathrm{T} 2$. Indeed, if $\mathrm{T} 1$ or $\mathrm{T} 2$ contain no solution, the classes in rows 4-7 or columns 4-7 of Table 1 can be directly discarded.

The second step is then attempting to discard the remaining rows and columns of Table 1 using systems T3 to T8. If any of the systems T3-T6 has no solution, the singularities in the corresponding row or column can not be possible. Discarding a row or a column may imply that other rows or columns are also not possible. For instance, if row 5 or 6 is discarded, row 7 is also discarded automatically because it includes the previous combination of singularity types. Row 7 only needs to be tested if both rows 5 and 6 have not been discarded. In such case, the test will be formed by the combination of the equations in T3 and T4. The same applies for columns 5-7 and tests T5 and T6. Only if the RPM- or IIM-types have been discarded in the first step, then T7, $\mathrm{T} 8$ and, eventually, the combination of $\mathrm{T} 7$ and $\mathrm{T} 8$ can be used to discard the remaining rows or columns.

If necessary, the third step follows by testing particular classes that have still not been discarded. To do so, combinations of the tests given in Table 2 must be used. The system of equations to test the (RO, RPM, II, IIM) singularity class (row 6 , column 6 of Table 1) would be formed by the equations in T4 and T5, for instance. Or, the test for the occurrence of the (RI, RO, RPM, IO, II, IIM) class (row 7, column 7 of Table 1) would be formed by the equations in $\mathrm{T} 3$ to $\mathrm{T} 6$.

It is important to notice that, no matter how small the resolution $\sigma$ of the method is chosen, each box will always contain a continuum of singular configurations, except for the case of isolated singular configurations. Thus, the process will be unable to precisely classify a given box when all singular configurations inside such a box are not all in the same singularity class. In rare cases, when a box contains configurations in at least four singularity types, the exact singularity class of the box configurations cannot be determined. Instead, a set of possible singularity classes is identified. Usually, such a box will contain at least two different singularity classes. For example, it may be known for a box that it contains singularities of types RI, RO, II, IO, and so it can be concluded that it contains either (a) configurations belonging to all four types or (b) a configuration in class (RI, IO) and another in (RO, II) or (c) configurations in all three classes. If there are no (RO, RI, IO, II) configurations in the box, this can be confirmed with further splitting. As illustrated by the following example, for most of the boxes only the unique class it intersects is determined unambiguously.

\section{AN ILLUSTRATIVE EXAMPLE}

To illustrate the process of the classification of the singularity set, the 2-dof planar manipulator shown in Fig. 3 is used. The inputs are the joint velocities at $A$ and $E$, and the output is the 
motion of point $G$. The equations $\boldsymbol{\Phi}(\boldsymbol{q})=0$ are written as

$$
\left\{\begin{array}{l}
0=-x+2 \cos \theta_{D}+\frac{3}{2} \cos \theta_{C} \\
0=-y+2 \sin \theta_{D}+\frac{3}{2} \sin \theta_{C} \\
0=\cos \theta_{A}+\cos \theta_{B}-2 \cos \theta_{D}-1 \\
0=\sin \theta_{A}+\sin \theta_{B}-2 \sin \theta_{D} \\
0=2 \cos \theta_{D}+\frac{3}{2} \cos \theta_{C}+2 \cos \theta_{G}-3 \cos \theta_{E}-1 \\
0=2 \sin \theta_{D}+\frac{3}{2} \sin \theta_{C}+2 \sin \theta_{G}-3 \sin \theta_{E}
\end{array}\right.
$$

where $\theta_{A}, \theta_{B}, \theta_{C}, \theta_{D}, \theta_{E}$ and $\theta_{G}$ are the counterclockwise angles of links $A B, B C, C G, D C, E F$, and $G F$, respectively, relative to the ground, and $x, y$ are the coordinates of point $G$ relative to a fixed frame centered in $D$. The velocity equation of the manipulator may be obtained, for instance, by differentiating (5) with respect to all variables, but it could also be obtained using the twist loop equations, or by any other means. In order to achieve the desired quadratic formulation for all systems in Eq. (2) and Table 2, the changes of variables $c_{\tau}=\cos \theta_{\tau}$ and $s_{\tau}=\sin \theta_{\tau}$ can be applied for all $\tau \in\{A, B, C, D, E, G\}$. Since the variables $c_{\tau}$ and $s_{\tau}$ represent the cosine and sine of a variable, the circle equations $c_{\tau}^{2}+s_{\tau}^{2}=1$ are introduced in the systems for all angles.

The manipulator can be seen as made of a 4-bar manipulator (loop $A B C D$ ) and a 5-bar manipulator (loop $D C G F E$ ) with one link in common $(C D)$. As a whole, the manipulator has two degrees of freedom, so its configuration space is a surface, and one may expect the singularity set to be made of curves or isolated configurations. However, the link dimensions are chosen so that the 4-bar part of the manipulator has a one-dimensional set of singular configurations (joint $C$ coincident with $A$, links $A B$ and $B C$ can rotate around $A$ ), while the other part can still move for each of those configurations, with $G$ moving on a circle. This gives raise to a two-dimensional subset of singularities inside the whole singularity set, which is illustrated in Fig. 4a, projected onto the $x, y$ and $\theta_{A}$ variables. The green, cyan and purple curves

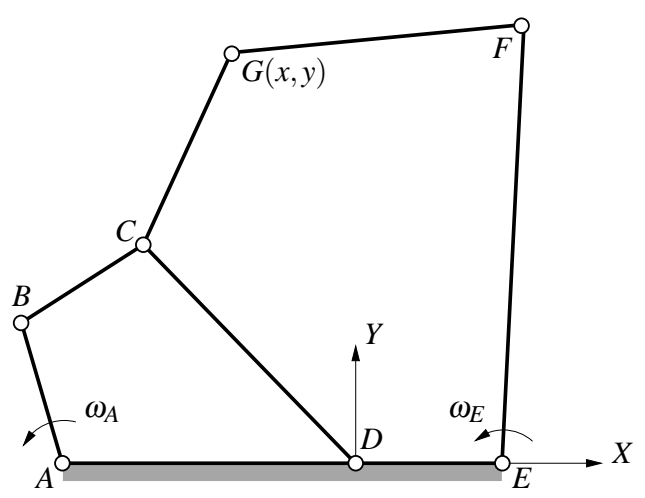

FIGURE 3. A 2-dof planar manipulator. The link dimensions are $A B=B C=D E=1, A D=C D=F G=2, C G=1.5$ and $E F=3$.

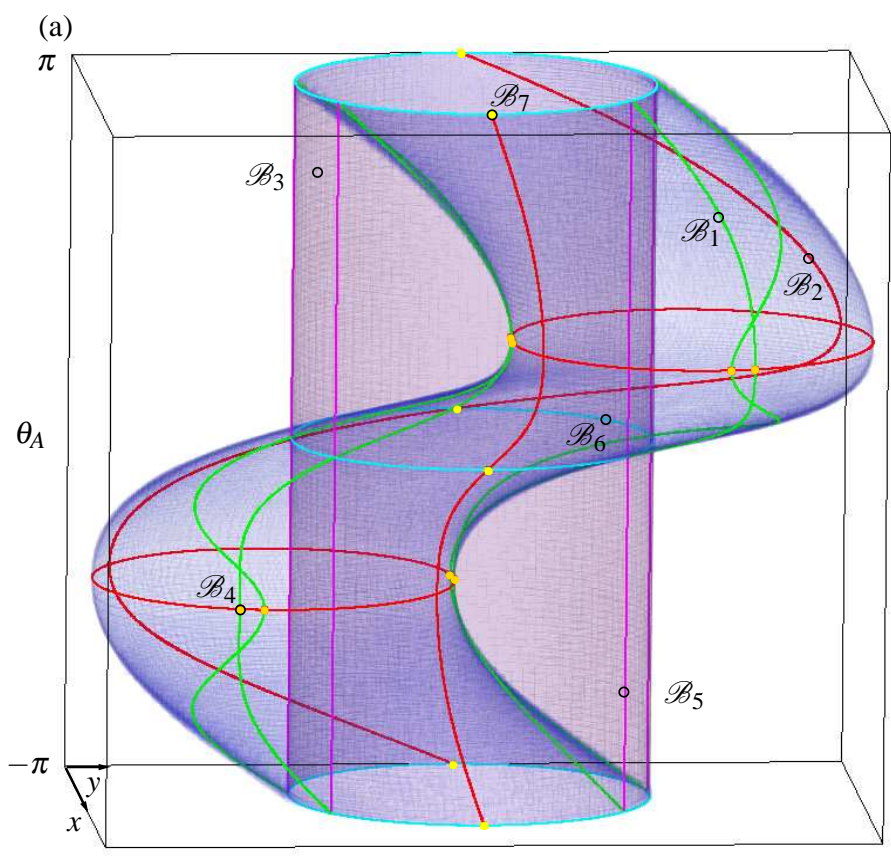

(b)

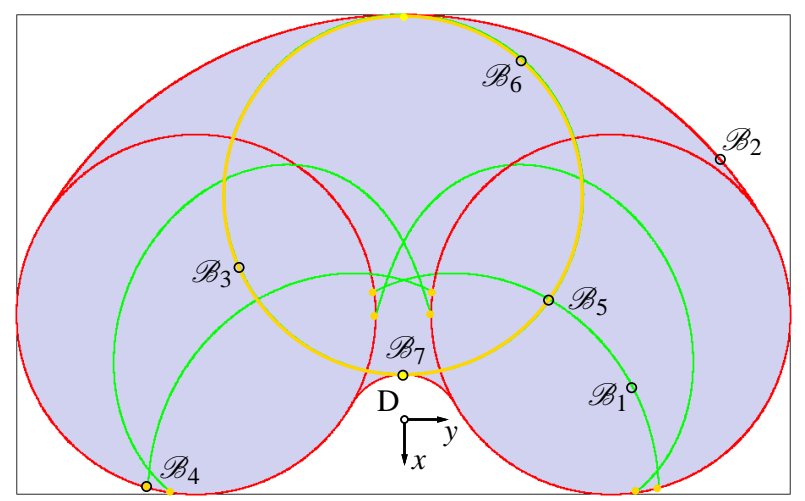

FIGURE 4. Complete singularity set projected onto the space of the $x, y$ and $\theta_{A}$ variables (top), and onto the space of the $x$ and $y$ variables only (bottom). The configuration space is shown in blue. Green curves: (RO, II), red curves and cylinder-shaped red surface: (RI, IO), orange dots: (RI, IO), (RO-II) and (RI-RO-II-IO), purple curves: (RI, IO) and (RI-RO-II-IO), cyan curves: (RI, IO) and (RI, RO, IIM), yellow dots: (RI, IO), (RI, RO, IIM) and (RI, RPM, IO, IIM).

are those configurations where the FIKP is undeterminate, and the red curves, together with the cylinder-shaped red surface, where the IIKP is undeterminate. Altogether, around 128000 boxes obtained by solving Eq. (2) at a small-enough $\sigma$, form an envelope of the singularity set of the manipulator. The surface of the configuration space is also shown in blue, obtained from the computation of all solutions of (1) using the same numerical technique. For clarity, the projection onto the $x$ and $y$ variables only is also shown in Fig. 4b, where the two-dimensional subset 


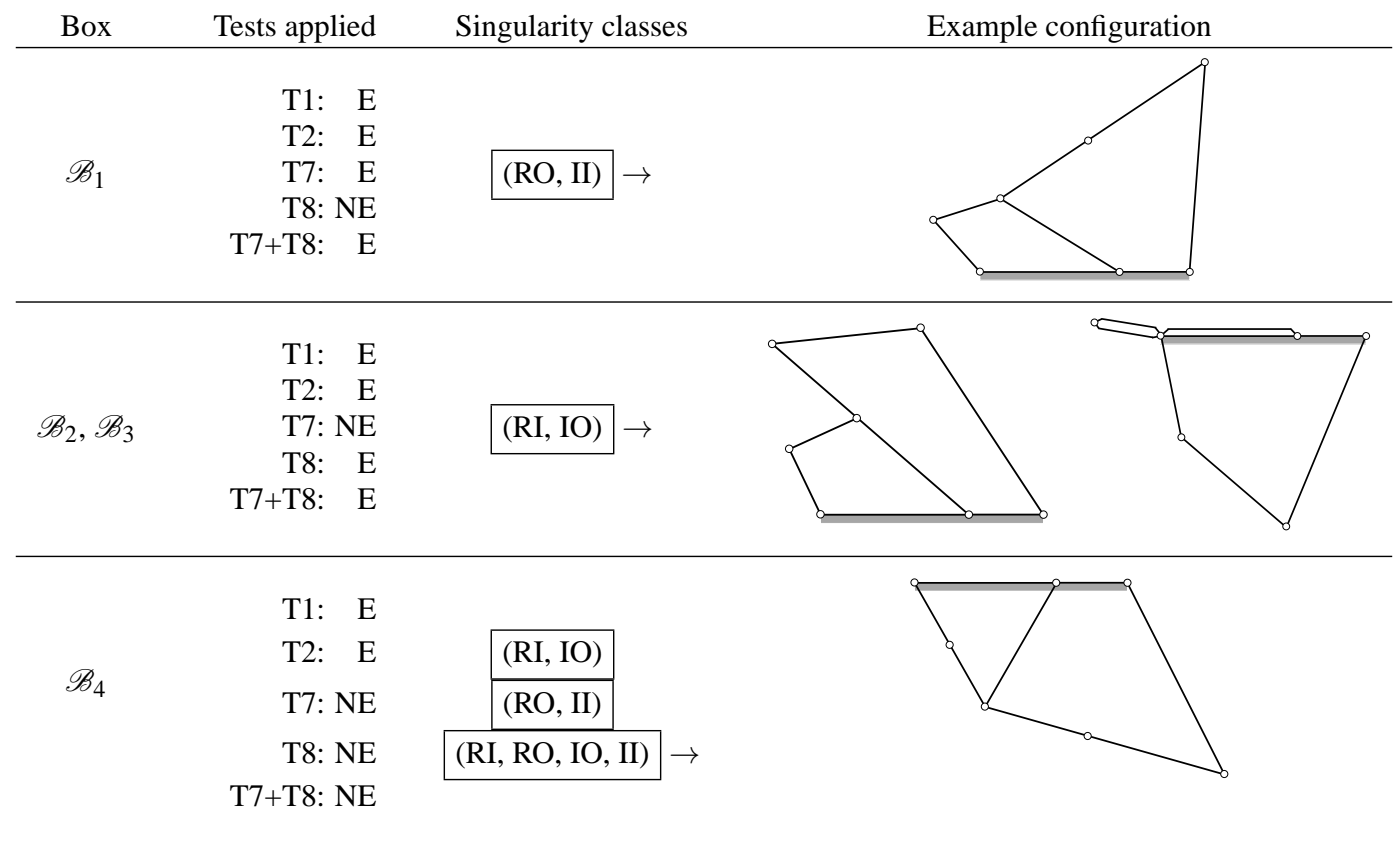

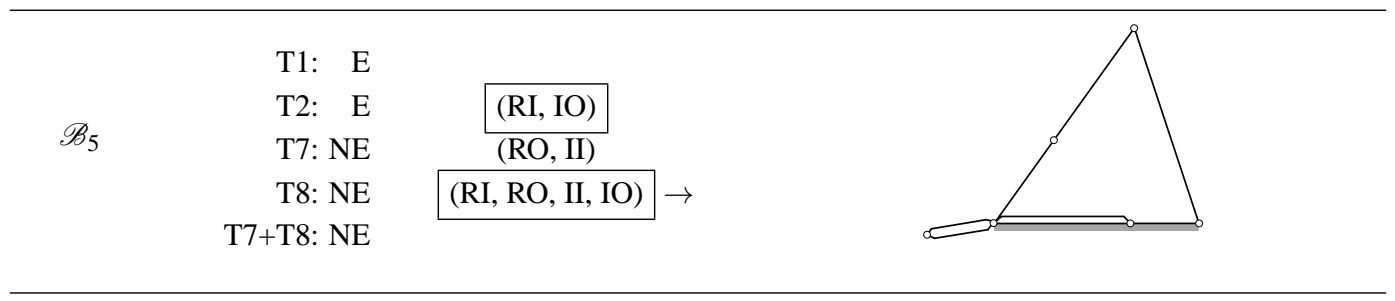

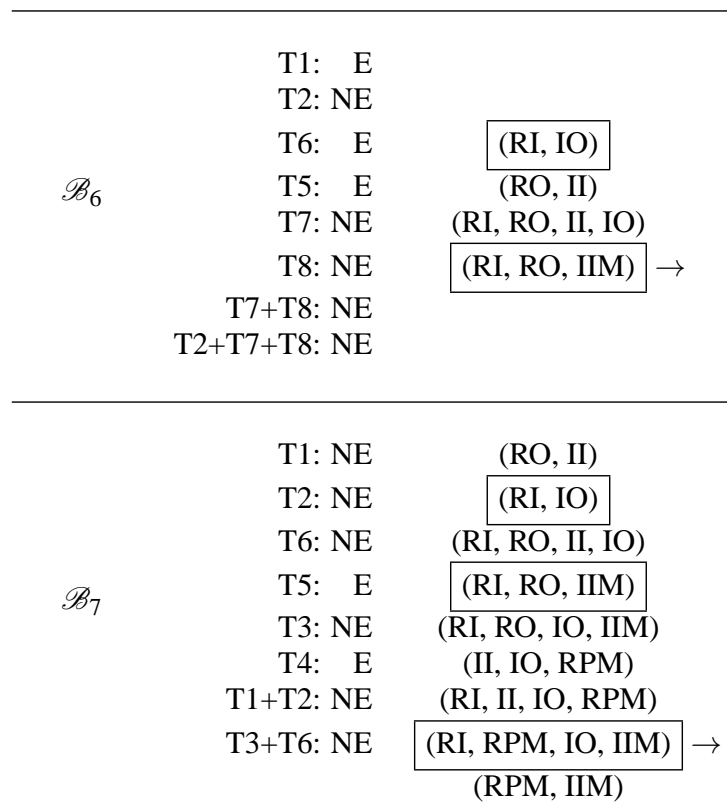

TABLE 3. Some example boxes of the process of classification. Column "Singularity classes" shows those classes that have not been discarded by the sequence of tests (column "Tests applied"), and those actually present inside the box are framed. Last column shows an example of a configuration in the box belonging to the singularity class indicated by an arrow. 
of singularities is projected to the orange circle.

During the whole process of classification, only 7 different kinds of boxes are found. To exemplify the classification, we will use an example box for each of these kinds, labelled $\mathscr{B}_{1}$ to $\mathscr{B}_{7}$ in Fig. 4. These can be defined as follows:

$\mathscr{B}_{1}$ : boxes where only the FIKP is undeterminate (green curves).

$\mathscr{B}_{2}$ : boxes where only the IIKP is undeterminate for $\theta_{D} \neq$ $\{-\pi, \pi\}$ (red curves).

$\mathscr{B}_{3}$ : boxes where only the IIKP is undeterminate for $\theta_{D}=$ $\{-\pi, \pi\}$ (red surface).

$\mathscr{B}_{4}$ : boxes where both the FIKP and IIKP are undeterminate and $\theta_{D} \neq\{-\pi, \pi\}$ (orange dots).

$\mathscr{B}_{5}$ : boxes where both the FIKP and IIKP are undeterminate for $\theta_{D}=\{-\pi, \pi\}$ and $\theta_{A} \neq\{0, \pi,-\pi\}$ (purple curves).

$\mathscr{B}_{6}$ : boxes where both the FIKP and IIKP are undeterminate for $\theta_{D}=\{-\pi, \pi\}, \theta_{A}=\{0, \pi,-\pi\}$ and $y \neq 0$ (cyan curves). $\mathscr{B}_{7}$ : boxes where both the FIKP and IIKP are undeterminate for $\theta_{D}=\{-\pi, \pi\}, \theta_{A}=\{0, \pi,-\pi\}$ and $y=0$ (yellow dots).

The result of the sequence of tests applied on these boxes can be seen in Table 3, where "E" and "NE" mean that the corresponding test returns an empty or a non-empty collection of boxes, respectively, and " $\mathrm{T} 7+\mathrm{T} 8$ ", for instance, indicates that the combination of the equations of $\mathrm{T} 7$ and $\mathrm{T} 8$ is used as test.

Most of the boxes correspond to the kinds of $\mathscr{B}_{1}, \mathscr{B}_{2}$ or $\mathscr{B}_{3}$, that get completely classified, and only in the intersection between singularity curves or between singularity curves and the singularity surface, other classes of singularities appear. For boxes of the kind $\mathscr{B}_{4}$ to $\mathscr{B}_{7}$, the process of classification leaves several possible singularity classes inside the box, but further inspection allows to discard some of them. In this example only 8 boxes correspond to $\mathscr{B}_{4}$, and they must contain configurations belonging to the (RI, IO), (RO, II) and (RI, RO, II, IO) classes, because no RPM- or IIM-types are present. In the case of $\mathscr{B}_{5}$, the purple curve is always on the red surface, so the (RO, II) class can be discarded. Looking at the classes that are not discarded by the tests in $\mathscr{B}_{6}$, it is straightforward that it must contain the class (RI, RO, IIM), and also (RI, IO), because it contains configurations on the red surface only. Similarly as for $\mathscr{B}_{5}$, the cyan curves are on the red surface, so the rest of classes can be discarded. Similar reasonings can be applied to the boxes corresponding to $\mathscr{B}_{7}$ to see that they only contain the (RI, IO), (RI, RO, IIM) and (RI, RPM, IO, IIM) classes.

As shown, most of the boxes that form the envelope of the singularity set of the manipulator get completely classified, and for those with several options, simple reasoning has allowed us to achieve a complete classification.

\section{CONCLUSIONS}

This article has presented an approach to obtain a complete description of the singularity set of any given non-redundant manipulator, including the identification and the precise computation of each constituent singularity class. This is specially useful and relevant in the early stages of the design of the manipulator, to identify the exact physical phenomena that occur at singular configurations, but also to study the behaviour of the mechanism at special configurations. The approach, based on a recent numerical method using linear relaxations, consists in applying a hierarchy of tests on the whole singularity set of the manipulator, computed using the same numerical technique, which yields sets of classes to which an identified singularity cannot belong. The procedure is able to completely classify most of the singularities, and only in some particular cases it identifies a restricted list of possible singularity classes. Further visual inspection of the singularity set, or building up new systems by combining the ones presented in this paper, may allow to discard some of the classes. For manipulators with more than 3 degrees of freedom, computation times may increase and visualization becomes difficult, as is the case with any method, but it is possible to consider lower-dimensional sections of the configuration space to circumvent this issue. The evaluation of the performance of the method on spatial parallel manipulators will be part of our future work.

\section{REFERENCES}

[1] Hunt, K., 1978. Kinematic Geometry of Mechanisms. Oxford University Press.

[2] Gosselin, C., 1990. "Determination of the workspace of 6DOF parallel manipulators". ASME Journal of Mechanical Design, 112, p. 331.

[3] Merlet, J. P., 1992. "Geometrical determination of the workspace of a constrained parallel manipulator". In Advances in Robot Kinematics, pp. 326-329.

[4] Merlet, J. P., Gosselin, C. M., and Mouly, N., 1998. "Workspaces of planar parallel manipulators". Mechanism and Machine Theory, 33(1-2), pp. 7-20.

[5] Merlet, J.-P., 1999. "Determination of 6D workspaces of Gough-type parallel manipulator and comparison between different geometries". International Journal of Robotics Research, 18(9), pp. 902-916.

[6] Bonev, I., and Ryu, J., 2001. "A new approach to orientation workspace analysis of 6-DOF parallel manipulators". Mechanism and Machine Theory, 36(1), pp. 15-28.

[7] Snyman, J. A., du Plessis, L. J., and Duffy, J., 2000. "An optimization approach to the determination of the boundaries of manipulator workspaces". ASME Journal of Mechanical Design, 122(4), pp. 447-456.

[8] Bonev, I. A., and Gosselin, C. M., 2006. "Analytical determination of the workspace of symmetrical spherical paral- 
lel mechanisms". IEEE Transactions on Robotics, 22(5), pp. 1011-1017.

[9] Zein, M., Wenger, P., and Chablat, D., 2006. "An exhaustive study of the workspace topologies of all 3R orthogonal manipulators with geometric simplifications". Mechanism and Machine Theory, 41(8), pp. 971-986.

[10] Ottaviano, E., Husty, M., and Ceccarelli, M., 2006. "Identification of the workspace boundary of a general 3-R manipulator". ASME Journal of Mechanical Design, 128(1), pp. 236-242.

[11] Haug, E. J., Luh, C.-M., Adkins, F. A., and Wang, J.-Y., 1996. "Numerical algorithms for mapping boundaries of manipulator workspaces". ASME Journal of Mechanical Design, 118, pp. 228-234.

[12] Li, H., Gosselin, C., Richard, M., and St-Onge, B., 2006. "Analytic form of the six-dimensional singularity locus of the general Gough-Stewart platform". ASME Journal of Mechanical Design, 128, pp. 279-288.

[13] Merlet, J.-P., 2007. "A formal-numerical approach for robust in-workspace singularity detection". IEEE Transactions on Robotics, 23(3), pp. 393-402.

[14] Jiang, Q., and Gosselin, C., 2009. "Determination of the maximal singularity-free orientation workspace for the Gough-Stewart platform". Mechanism and Machine Theory, 44(6), pp. 1281-1293.

[15] Gosselin, C., and Angeles, J., 1990. "Singularity analysis of closed-loop kinematic chains". IEEE Transactions on Robotics and Automation, 6, pp. 281-290.

[16] Park, F., and Kim, J., 1996. "Manipulability of closed kinematic chains". ASME Journal of Mechanical Design, 120, pp. 542-548.

[17] Park, F., and Kim, J., 1999. "Singularity analysis of closed kinematic chains". ASME Journal of Mechanical Design, 121, pp. 32-38.

[18] Zlatanov, D., Fenton, R., and Benhabib, B., 1995. "A unifying framework for classification and interpretation of mechanism singularities". ASME Journal of Mechanical Design, 117 , pp. 566-572.

[19] Zlatanov, D., 1998. "Generalized singularity analysis of mechanisms". $\mathrm{PhD}$ thesis, University of Toronto.

[20] Zlatanov, D., Benhabib, B., and Fenton, R., 1998. "Identification and classification of the singular configurations of mechanisms". Mechanism and Machine Theory, 33, pp. 743-760.

[21] Bohigas, O., Zlatanov, D., Ros, L., Manubens, M., and Porta, J. M., 2012. "Numerical computation of manipulator singularities". In Proc. of the IEEE Int. Conf. on Robotics and Automation. To appear.

[22] De Jalón, J. G., and Bayo, E., 1993. Kinematic and Dynamic Simulation of Multibody Systems. Springer Verlag.

[23] Selig, J., 2005. Geometric fundamentals of robotics. Springer.
[24] Porta, J. M., Ros, L., and Thomas, F., 2009. "A linear relaxation technique for the position analysis of multi-loop linkages". IEEE Transactions on Robotics, 25(2), pp. 225239. 